\title{
Topological defects and open inflation
}

\author{
Alexander Vilenkin \\ Institute of Cosmology, Department of Physics and Astronomy, \\ Tufts University, Medford, MA 02155, USA
}

Topological defects can naturally be formed soon after bubble nucleation in the open inflation scenario. The defects are not completely diluted away by the subsequent period of inflation in the bubble interior and can produce observable large-scale microwave background anisotropies. Superheavy strings and monopoles attached to the strings can act as gravitational lenses with angular separation between the images of up to an arc minute.

\section{INTRODUCTION}

Until recently, a flat universe with $\Omega=1$ was regarded as a firm prediction of inflationary models. However, the observational evidence for a flat universe is far from being clear, and a new class of models called "open inflation" has recently become a subject of active investigation [1 [3]. In these models, the visible universe is contained within a single bubble which nucleated in an inflating false vacuum. The observed homogeneity of the universe is ensured by a high symmetry of the bubble, rather than by a large expansion factor, as in the more familiar inflation models $[4$. After nucleation, inflation continues for some time in the bubble interior, and the corresponding number of e-foldings can be adjusted to give any value of the density parameter $\Omega$ between $\Omega=0$ and $\Omega=1$.

The first models of open inflation [1.2] gave a fixed value of $\Omega$, and obtaining a value not too close to 0 or 1 required a substantial amount of fine-tuning. A significant improvement was made by Linde and Mezhlumian [3] who suggested a number of models where $\Omega$ is a continuous parameter taking different values in different bubbles. The 
probability distribution for $\Omega$ in such models was discussed in Ref. [5], where it was argued that, with the anthropic factor properly taken into account, this distribution can naturally be peaked at an intermediate value of $\Omega$. Open inflation may thus require no more fine-tuning than 'ordinary' inflation.

The purpose of this paper is to make a simple observation that, in models of open inflation, topological defects are likely to be formed soon after the bubble nucleation. The defects will not be completely diluted away by the short period of inflation in the bubble interior, and we may still be able to observe them on very large scales comparable to the curvature scale of the universe.

\section{DEFECT FORMATION AND EVOLUTION}

The models suggested by Linde and Mezhlumian involve two scalar fields, $\phi_{1}$ responsible for bubble nucleation and $\phi_{2}$ responsible for the slow-roll inflation inside the bubble. For a specific example consider the model with a potential [3]

$$
U\left(\phi_{1}, \phi_{2}\right)=V\left(\phi_{1}\right)+\lambda \phi_{1}^{2} \phi_{2}^{2}
$$

where $V\left(\phi_{1}\right)$ has a metastable minimum at $\phi_{1}=0$ and the true minimum at $\phi_{1}=\eta_{1}$. The stage for open inflation is set by the inflating false vacuum at $\phi_{1}=0$. The corresponding energy density is $\rho_{1}=V(0)$, and the spacetime is nearly de Sitter with an expansion rate $H_{1}=\left(8 \pi \rho_{1} / 3 m_{p}^{2}\right)^{1 / 2}$, where $m_{p}$ is the Planck mass. Bubble nucleation can occur to a wide range of values of the field $\phi_{2}$. The second stage of inflation takes place while $\phi_{2}$ rolls towards its minimum at $\phi_{2}=0$, and the resulting density parameter $\Omega$ is determined by the initial value of $\phi_{2}$ at the time of bubble nucleation.

The metric in the bubble interior is well approximated by the Robertson-Walker (RW) metric

$$
d s^{2}=d t^{2}-a^{2}(t)\left[d \zeta^{2}+\sinh ^{2} \zeta\left(d \theta^{2}+\sin ^{2} \theta d \phi^{2}\right)\right]
$$


with $a(t)$ satisfying

$$
\dot{a}^{2}-1=\left(8 \pi / 3 m_{p}^{2}\right) \rho a^{2} .
$$

Here, $\rho$ is the energy density measured by co-moving RW observers. The hypersurface $t=0$, where $a=0$, is the future light cone of the bubble center at the moment of nucleation. The coordinate system (2) has a singularity on this surface, but the fourgeometry is of course non-singular.

For a generic shape of the potential $V\left(\phi_{1}\right)$, the initial radius of the bubble $R$ is comparable to the thickness of the bubble wall. At $t \sim R$, when $a(R) \sim R$ and $\rho \sim \rho_{1}$, the field $\phi_{1}$ begins to oscillate about $\phi_{1}=\eta_{1}$, and at later times its energy density scales like that of non-relativistic matter. The initial bubble radius cannot exceed the horizon, $R \lesssim H_{1}^{-1}$, and it is easily seen from Eq. (3) that the density term is either negligible from the very beginning or becomes negligible in a few Hubble times after the nucleation. Hence, the buble-universe is curvature-dominated, with $a(t) \approx t$ and $\rho \sim \rho_{1}(R / t)^{3}$.

The second period of inflation begins at $t \sim H_{2}^{-1}$, where $H_{2}=\left(8 \pi \rho_{2} / 3 m_{p}^{2}\right)^{1 / 2}, \rho_{2}=$ $\lambda \eta_{1}^{2} \phi_{2 i}^{2}$ and $\phi_{2 i}$ is the initial value of $\phi_{2}$ at nucleation. Between the nucleation and second inflation, the bubble-universe expands by a factor $f \sim\left(H_{2} R\right)^{-1} \gtrsim H_{1} / H_{2} \sim\left(\rho_{1} / \rho_{2}\right)^{1 / 2}$. The value of $\rho_{2}$ is constrained to be smaller than $\rho_{1}$, and since there is no reason for $\rho_{2}$ to be $\sim \rho_{1}$, we expect that generically $\rho_{2} \ll \rho_{1}$ and $f \gg 1$. The field $\phi_{2}$ evolves on a timescale $H_{2} / m_{2}^{2} \gg H_{2}^{-1}$ and remains essentially constant during the period between the two inflations. Here, $m_{2}=(2 \lambda)^{1 / 2} \eta_{1} \ll H_{2}$ is the mass of $\phi_{2}$.

Defect formation between inflations can be triggered by the same mechanisms as defect formation during inflation which has been previously discussed in the literature [6 9]. Let $\chi$ be the field responsible for the defects and let us first consider a model where $\chi$ has a non-minimal coupling to the curvature $\mathcal{R}$,

$$
L_{\chi}=\left|\partial_{\mu} \chi\right|^{2}+M^{2}|\chi|^{2}-\kappa \mathcal{R}|\chi|^{2}-\lambda_{\chi}|\chi|^{4}
$$


We shall assume for definiteness that $\chi$ is a complex scalar field. The effective mass squared of $\chi$ is

$$
m_{\chi}^{2}=-M^{2}+\kappa \mathcal{R}
$$

From Einstein's equations $\mathcal{R}=8 \pi \rho / m_{p}^{2}$, and thus $m_{\chi}^{2}$ changes sign at $\rho_{c}=M^{2} m_{p}^{2} / 8 \pi \kappa$. This triggers a symmetry-breaking phase transition resulting in the formation of cosmic strings with mass per unit length $\mu \sim M^{2} / \lambda_{\chi}$. The phase transition occurs between inflations provided that $\rho_{1}>\rho_{c}>\rho_{2}$ and $M>H_{2}$. These conditions require no fine tuning and can be naturally satisfied in a variety of models.

An alternative scenario is that the $\phi_{2}$ particles are unstable with a lifetime $\tau_{2}<H_{2}^{-1}$. Their decay products (call them $\sigma$ ) may then trigger the phase transition by a direct interaction with $\chi$, e.g., $L_{\chi \sigma}=-\lambda_{\chi \sigma} \sigma^{2}|\chi|^{2}$. If the decay products of $\phi_{2}$ have enough time to thermalize, then the corresponding phase transition is the 'usual', thermal phase transition.

Finally, there is one more mechanism which is specific to open inflation. Suppose now that $\chi$ is coupled to the tunneling field $\phi_{1}$,

$$
L_{\chi}=\left|\partial_{\mu} \chi\right|^{2}-M^{2}|\chi|^{2}+\lambda_{1} \phi_{1}^{2}|\chi|^{2}-\lambda_{\chi}|\chi|^{4}
$$

In the false vacuum, $\phi_{1}=0$ and $\chi=0$, while in the true vacuum $\chi$ has a non-zero expectation value. Now, there are two possibilities. If a bubble nucleates with a non-zero value of $\chi$, then this value will tend to be homogeneous throughout the bubble-universe, and no defects will be formed. Alternatively, $\chi=0$ at nucleation and defect formation does occur. The initial value of $\chi$ is determined by the instanton solution of the Euclidean field equations [10]. The four-geometry of this solution is compact, with a characteristic size $\sim H_{1}^{-1}$. If this size is smaller than the length scales characterizing the field $\chi, M^{-1}$ and $\lambda_{1}^{-1 / 2} \eta_{1}^{-1}$, then $\chi=0$ on the instanton. For $H_{1}>M>H_{2}$, the field $\chi$ initially 
remains near zero, and starts evolving at $t \sim M^{-1}$. This can be regarded as the time of string formation.

If strings are formed at $t_{i} \sim M^{-1}$, then the initial string separation is $\xi_{i} \sim M^{-1}$, which corresponds to a co-moving scale $\zeta \sim 1$ in the metric (2). In the course of the following evolution, $\xi$ is bounded from above by the horizon, $t$, and from below by $\left[a(t) / a\left(t_{i}\right)\right] \xi_{i}$. Since both of these bounds are $\sim t$, we have $\xi \sim t$ all the way until the beginning of the second inflation, $t \lesssim H_{2}^{-1}$. To be more precise, the horizon in metric (国) with $a(t) \propto t$ grows like $t \ln t$ and Spergel [1] has argued that the characteristic scale of defects will grow like

$$
\xi \propto t(\ln t)^{1 / 2}
$$

In most of the following discussion I disregard these logarithmic factors.

During inflation, the strings are "frozen", that is, they are conformally stretched with their co-moving scale remaining at $\zeta \sim 1$. They start moving again at $t_{*} \sim \Omega / H_{0}$ (or redshift $\left(1+z_{*}\right) \sim \Omega^{-1}$ ), when the curvature scale $\zeta \sim 1$ comes within the horizon. Here, $H_{0}$ is the value of the Hubble parameter, $H=\dot{a} / a$, at the present time. At $t>t_{*}$ the strings will evolve in a scaling regime with $\xi(t) \sim t$, and thus the co-moving scale of strings will remain comparable to the curvature scale. The growth of density fluctuations in an open universe ceases at $t>t_{*}$, and since the strings do not generate fluctuations at $t<t_{*}$, they clearly could not be responsible for structure formation. Yet, the mass scale of the strings can be quite high and they can produce some observable effects.

In models where the phase transition is triggered by the curvature or by the decay products of $\phi_{1}$, it is possible to have $\xi_{i} \ll t_{i}$. The strings will then evolve towards a scaling regime with $\xi(t) \sim t$, and for $t_{i} \ll H_{2}^{-1}$ we may still have $\xi \sim t$ by the beginning of second inflation. However, the timescale on which the string scale grows up to $t$ is very model-dependent. For example, strings may be overdamped due to their interaction with the decay products of $\phi_{1}$. In this case their evolution will be rather slow, and the 
co-moving scale of strings during inflation may be $\zeta \ll 1$. It is conceivable that this scale can be small enough for strings to play a role in structure formation.

Suppose now that the defects formed between inflations are magnetic monopoles. The typical co-moving separation of the monopoles is then $\zeta \sim 1$, and the resulting monopole density is totally negligible. However, the situation is drastically changed if the monopoles get connected by strings at a subsequent phase transition.

Strings can either be formed during inflation or in the postinflationary epoch. The characteristic length scale of string at formation is then much smaller than the monopole separation; the strings connecting monopoles have Brownian shapes, and there is a large number of closed loops. The evolution of strings after inflation is initially identical to that of topologically stable strings, without monopoles [9]. In the course of the evolution, the characteristic length of strings grows like $t$ and becomes comparable to the monopole separation at $t \sim t_{*}$, so that we are left with monopole-antimonopole pairs connected by more or less straight strings of length $\ell \sim t_{*}$. At $t>t_{*}$, the pairs oscillate and gradually lose their energy by gravitational or gauge boson radiation. The corresponding lifetimes are, respectively [12,13], $\tau_{g r} \sim t_{*} / \Gamma_{g r} G \mu$ and $\tau_{b} \sim \mu t_{*} / \alpha m^{2}$. Here, $\Gamma_{g r} \sim 10^{3}$ is a numerical coefficient, $\alpha \sim 10^{-2}$ is the gauge coupling constant, and $m$ is the monopole mass. Since the symmetry breaking scale of the monopoles is greater than that of the strings, we should have $m^{2} \gtrsim \mu$, and it is easily seen that both lifetimes $\tau_{g r}$ and $\tau_{b}$ are

much greater than the present age of the universe (provided that $\Omega \gtrsim 0.1$ ). Note that if monopoles and strings are formed after inflation, then the lifetime of the pairs is typically very short, and they decay well before the end of the radiation era.

\section{OBSERVATIONAL EFFECTS}

Let us first consider possible effects of 'plain' cosmic strings, without monopoles. Strings can produce double images of high-redshift galaxies and quasars [14]. The metric 
of a straight string in an open universe is given [16] by the same Eq.(2), but with a modified range for the angular variable $\phi, 0<\phi<2 \pi-\Delta$, where $\Delta=8 \pi G \mu$ is the deficit angle and $\mu$ is the mass per unit length of string. Let $\zeta_{O}$ and $\zeta_{G}$ be, respectively, the coordinate distances from the string to the observer and to the galaxy, and let $\theta$ be the angle between the string and the line of sight. Then it is easily shown that the angular separation between the images is

$$
\delta=8 \pi G \mu \frac{\sinh \zeta_{G}}{\sinh \left(\zeta_{G}+\zeta_{O}\right)} \sin \theta .
$$

Here, I have assumed for simplicity that the string is static and that $G \mu \ll 1$. For $\zeta_{G}, \zeta_{O} \ll 1$, Eq. (8) reduces to the expression for the angular separation in a spatially flat universe [14].

Moving strings induce discontinuous jumps in the microwave background temperature 17, 15,

$$
\delta T / T \sim 8 \pi \beta G \mu v
$$

where $v$ is the string velocity with respect to local co-moving observers and $\beta \sim 0.5$ is a trigonometric factor. At $t>t_{*}$, the universe becomes curvature-dominated and expands like $a(t) \propto t$. This regime is "on the verge" of inflation, in the sense that an expansion law $a(t) \propto t^{\alpha}$ with $\alpha>1$ corresponds to a power-law inflation. Since the strings are frozen during inflation, one can expect [18] their typical velocity in a curvature-dominated universe to be well below that in a radiation or matter-dominated universe $\left(v_{r} \sim v_{m} \sim 0.6\right)$.

The pattern of the microwave temperature fluctuations on the sky is a superposition of the contributions of strings from different redshifts between $z_{*} \sim \Omega^{-1}$ and the present. The highest density of strings corresponds to the largest redshifts near $z_{*}$, and the typical angular separation of discontinuities on the sky is $\theta_{\min } \sim \Omega$. We expect temperature fluctuations of magnitude (9) on scales $\theta_{\min } \lesssim \theta \lesssim 1$. To avoid conflict with observations, 
we should require $\delta T / T \lesssim 10^{-5}$. With $v \sim 0.2$ this gives a bound $G \mu \lesssim 10^{-5}$. According to Eq. (8), a string with $G \mu \sim 10^{-5}$ can give double images with angular separations of up to an arc minute.

Gravitational waves produced by the strings in this scenario will have too low frequencies to be detected by the existing methods, and thus the usual bounds on $G \mu$ from the millisecond pulsar observations and from nucleosynthesis considerations do not apply.

Note that if the logarithmic factor in Eq. (7) is indeed present, then the co-moving scale of strings at $t<t_{*}$ can be somewhat larger than the curvature scale. This will have the effect of moving $t_{*}$ closer to the present time and increasing the angle $\theta_{\min }$. In extreme cases, the typical string separation can even be greater than the present horizon.

Let us now turn to monopoles connected by strings. Monopoles are pulled by the strings with a force $\mu$ and accelerate to ultrarelativistic speeds. The typical energy of a monopole at time $t<t_{*}$ is $E \sim \mu t$, and at $t>t_{*}$ is $E \sim \mu t_{*}$. The latter corresponds to a mass

$$
\mu t_{*} \sim 10^{16}\left(\frac{G \mu}{10^{-6}}\right) \Omega M_{\odot}
$$

For grand-unification-scale strings with $G \mu \sim 10^{-6}$, this is comparable to the mass of a supercluster!

The microwave anisotropy produced by a moving point mass in Minkowski space has been calculated by Stebbins [19],

$$
\frac{\delta T}{T}=-\frac{4 G E v_{\perp}}{r \theta} \cos \alpha
$$

Here, $r$ is the distance from the observer to the point mass, $E=M\left(1-v^{2}\right)^{-1 / 2}$ is the total energy of the mass, $v$ is its velocity, $v_{\perp}$ is the velocity projected on the sky, $\theta$ is the angular distance between the mass $(M)$ and the point of observation $(P)$, and $\alpha$ is the angle between the line $M P$ and the projected velocity. It is assumed that $\theta \ll 1$. To extend this equation to the case of an expanding universe, we note that $r \theta=\ell_{\perp}$ is the 
closest-approach distance between the microwave photon and the mass. In a low-density universe, and for a mass at a redshift in the range $1 \lesssim z \lesssim z_{*}$, this relation is replaced by 20] $\theta=H_{0} \ell_{\perp} F(z)$, where $F(z) \approx 2$. Hence, we can write

$$
\delta T / T=8 G H_{0} E v_{\perp} \theta^{-1} \cos \alpha .
$$

With $E \sim \mu t_{*}$ and $v_{\perp} \sim 1$, this gives

$$
\delta T / T \sim 4 G \mu \Omega \theta^{-1}
$$

The density of monopoles with $z \lesssim z_{*}$ on the sky is dominated by the monopoles at $z \sim z_{*}$, so the typical angular separation between monopoles is $\theta \sim \Omega$. Monopoles at $z>z_{*}$ have smaller energies, and the corresponding microwave anisotropies are proportionally weaker. For $G \mu \sim 10^{-6}$ and $\Omega \gtrsim 0.1$, the anisotropies produced by moving monopoles have a detectable magnitude $\delta T / T \gtrsim 10^{-5}$ up to the angular distance of a few degrees.

Ultrarelativistic monopoles can also act as gravitational lenses. If the monopole and the galaxy which is being lensed are both at $z \sim 1$, then the typical light deflection angle is $\theta \sim G E / \ell_{\perp}$. Since $\ell_{\perp} \sim \theta H_{0}^{-1}$ and $E \sim \mu t_{*}$, we obtain an estimate for the typical angular separation between the images,

$$
\delta \phi \sim(G \mu \Omega)^{1 / 2}
$$

For $G \mu \sim 10^{-6}$ this separation is about an arc minute.

Global monopoles and textures produced between inflations will generate microwave background anisotropies, and global monopoles can also act as gravitational lenses. The evolution of global defects in an open universe and their effect on the microwave background have been studied by Pen and Spergel [18]. A bound on the symmetry breaking scale of defects can probably be extracted from their results, but I was unable to do so.

Finally, I would like to emphasize that, apart from defect formation between inflations, some topological defects can be formed during or after inflation. The characteristic 
length scale of such defects is much smaller than the curvature scale, and they may be suitable as seeds for structure formation. Defect-seeded structure formation in an open universe has been discussed in Refs. [18,22].

I am grateful to David Spergel and Albert Stebbins for useful correspondence and to Martin Bucher and Andrei Linde for their comments on the paper. This work was supported in part by the National Science Foundation.

[1] J.R. Gott, Nature 295, 304 (1982).

[2] M. Bucher, A.S. Goldhaber and N. Turok, Phys. Rev. D55, 3314 (1995); K. Yamamoto, M. Sasaki and T. Tanaka, Ap. J. 455, 412 (1995).

[3] A.D. Linde, Phys. Lett. B351, 99 (1995); A.D. Linde and A. Mezhlumian, Phys. Rev. D52, 6789 (1995).

[4] For a review of inflation see A.D. Linde, Particle Physics and Inflationary Cosmology (Harwood, Chur, Switzerland, 1990); K.A. Olive, Phys. Rep. 190, 307 (1990).

[5] A. Vilenkin and S. Winitzki, Phys. Rev. D55, 548 (1997).

[6] Q. Shafi and A. Vilenkin, Phys. Rev. D29, 1870 (1984).

[7] L. Kofman and A.D. Linde, Nucl. Phys. B282, 555 (1987).

[8] E.T. Vishniak, K.A. Olive and D. Seckel, Nucl. Phys. B289, 717 (1987).

[9] For a review of topological defects in cosmology, see M.B. Hindmarsh and T.W.B. Kibble, Rep. Prog. Phys. 55, 478 (1995); A. Vilenkin and E.P.S. Shellard, Cosmic Strings and Other Topological Defects (Cambridge University Press, Cambridge, 1994).

[10] S. Coleman and F. de Luccia, Phys. Rev. D21, 3305 (1980). 
[11] D.N. Spergel, Ap. J. Lett. 412, 5 (1993).

[12] X. Martin and A. Vilenkin, Phys. Rev. Lett. 77, 2879 (1996); Phys. Rev. D, in press gr$\mathrm{qc} / 9612008$.

[13] V. Berezinsky, X. Martin and A. Vilenkin, unpublished astro-ph/9703077.

[14] A. Vilenkin, Phys. Rev. D23, 852 (1981); Ap. J. Lett. 51, 282 (1984).

[15] J.R. Gott, Ap. J. 288, 422 (1985).

[16] M. Aryal, L.H. Ford and A. Vilenkin, Phys. Rev. D34, 2263 (1986).

[17] N. Kaiser and A. Stebbins, Nature 310, 391 (1984).

[18] D.D. Spergel and U. Pen, Phys. Rev. D51, 4099 (1995).

[19] A. Stebbins and S. Veeraraghavan, Phys. Rev. D51, 1465 (1995).

[20] See, e.g., Eq. (13.47) and Fig. 13.5 of Peebles' book 21].

[21] P.J.E. Peebles, Principles of Physical Cosmology (Princeton University Press, Princeton, 1993).

[22] P. Ferreira, Phys. Rev. Lett. 74, 3522 (1995). 